\title{
Origin of elemental carbon in snow from Western Siberia and northwestern European Russia during winter-spring 2014, 2015 and 2016
}

Nikolaos Evangeliou ${ }^{1, *}$, Vladimir P. Shevchenko ${ }^{2}$, Karl Espen Yttri ${ }^{1}$, Sabine Eckhardt ${ }^{1}$, Espen Sollum ${ }^{1}$, Oleg S. Pokrovsky ${ }^{3,4}$, Vasily O. Kobelev ${ }^{5}$, Vladimir B. Korobov $^{6}$, Andrey A. Lobanov ${ }^{5}$, Dina P. Starodymova ${ }^{2}$, Sergey N. Vorobiev $^{7}$, Rona L. Thompson ${ }^{1}$, Andreas Stohl ${ }^{1}$

${ }^{1}$ NILU - Norwegian Institute for Air Research, Department of Atmospheric and Climate Research (ATMOS), Kjeller, Norway.

${ }^{2}$ Shirshov Institute of Oceanology, Russian Academy of Sciences, Nakhimovsky prospect 36, 117997 Moscow, Russia.

${ }^{3}$ Geosciences Environment Toulouse, UMR 5563 CNRS, University of Toulouse, 14 Avenue Edouard Belin, 31400, Toulouse, France.

${ }^{4}$ N. Laverov Federal Center for Integrated Arctic Research, Russian Academy of Science, Sadovaya street, 3, 163000, Arkhangelsk, Russia.

${ }^{5}$ Arctic Research Center of the Yamalo-Nenets autonomous district, Vos'moy proezd, NZIA building, 629730, Nadym, Yamalo-Nenets autonomous district, Russia.

${ }^{6}$ North-Western Brunch of Shirshov Institute of Oceanology, Russian Academy of Sciences, Naberezhnaya Severnoy Dviny 112/3, 163061, Arkhangelsk, Russia.

${ }^{7}$ BIO-GEO-CLIM Laboratory, Tomsk State University, 36 Prospect Lenina, 634050, Tomsk, Russia.

\footnotetext{
*Correspondence to: N. Evangeliou, NILU - Norwegian Institute for Air Research, Department of Atmospheric and Climate Research (ATMOS), Kjeller, Norway (Nikolaos.Evangeliou@nilu.no)
} 


\section{Quality assurance of the EC measurements}

Minerals, such as calcite $\left(\mathrm{CaCO}_{3}\right)$ and dolomite $\left[\mathrm{CaMg}\left(\mathrm{CO}_{3}\right)\right]$, contain $\mathrm{CO}_{3}^{2-}$ - carbon, which can be detected by the thermal-optical measurement principle. Depending on the chemical speciation, $\mathrm{CO}_{3}^{2-}$ - carbon will evolve over a broad range of temperatures (Cavalli et al. 2010; Gunasekaran \& Anbalagan 2007), which coincides with the temperature range used in thermal-optical analysis (TOA). Whether $\mathrm{CO}_{3}^{2-}$ - carbon evolves as part of the organic carbon (OC) or the elemental carbon (EC) fraction, or both, depends on the protocol applied, but this question is still not fully explored even for the most commonly used protocols (NIOSH; IMPROVE; EUSAAR-2). Typically, acid fumigation has been used prior to analysis to eliminate and/or quantify the $\mathrm{CO}_{3}^{2-}$ - carbon contribution to the $\mathrm{OC}$ and/or EC signal (Chow et al., 1993). However, this approach can cause loss of volatile organic acids (Chow et al., 1993), induce severe charring (Jankowski et al. 2008), and importantly, the time of $\mathrm{CO}_{3}^{2-}$ carbon evolving is defined prior to the analysis. Experience based on the thermal-oxidative pretreatment approach (Jankowski et al. 2008), and subsequent analysis by the EUSAAR-2 protocol (as used in the current study) of ambient aerosol filter samples from Eastern Europe, Caucasus and Central Asia (the EECAA countries) and from the Arabian Peninsula, suggests that $\mathrm{CO}_{3}^{2-}$ - carbon evolves both as OC and EC. A similar finding is made for the filtered snow samples in the current study. The results presented in Table S 2 indicate a minor influence of $\mathrm{CO}_{3}^{2-}$ - carbon evolving as EC for samples collected in 2015 and 2016, whereas it was a factor of $2-4$ times higher for the samples collected in 2014. For a few samples though, $\mathrm{CO}_{3}^{2-}$ - carbon made a substantial ( $\left.>50 \%\right)$ contribution to the EC signal.

The thermal-oxidative pre-treatment approach coupled with the EUSAAR-2 protocol applied in the current study, has been demonstrated to provide similar results as that of the acid fumigation approach, with respect to concentrations of $\mathrm{CO}_{3}^{2-}$ - carbon from calcite (Fagerli et al. 2015). This is successful in removing OC and EC, but not carbonate carbon, during the thermal-oxidative pre-treatment step.

All filter samples in the current data set had an EC level of $<15 \mu \mathrm{g} \mathrm{C} \mathrm{cm}^{-2}$, which is considered the upper limit when analysing filter samples by TOA (Subramanian $\uparrow$ et al. 2006; Wallén et al. 2010). The mean $E C_{\mathrm{CO}_{3}^{2-}}^{\mathrm{corr}}-\mathrm{TC}_{\mathrm{CO}_{3}^{2-}}^{\mathrm{corr}}$ ratio of the filtered snow samples ranged from $0.031-0.091$, which is in the lowest range of what has been observed for atmospheric aerosol particles in the Northern European rural background environment (Yttri et al. 2007). Albeit crude, EC is considered as a tracer of anthropogenic activity; however, the very low 
$E C_{\mathrm{CO}_{3}^{2-}}^{\mathrm{corr}}-\mathrm{TC}_{\mathrm{CO}_{3}^{2-}}^{\mathrm{corr}}$ ratio cannot be used as an argument of low anthropogenic influence, as there can be non-atmospheric sources contributing to $\mathrm{TC}_{\mathrm{CO}_{3}^{2-}}^{\mathrm{Corr}}$. 
Table S 1. Information about the samples collected in springtime of 2014, 2015 and 2016 in Western Russia.

\begin{tabular}{lllllllll}
\hline Latitude & Longitude & Day & Month & Year & $\begin{array}{l}\text { Snow depth } \\
(\mathrm{m})\end{array}$ & $\begin{array}{l}\text { Surface sampled } \\
\left(\mathrm{m}^{2}\right)\end{array}$ & $\begin{array}{l}\text { Snow } \\
\text { Volume }(\mathrm{L})\end{array}$ & $\begin{array}{l}\text { Water equivalent } \\
\text { volume (L) }\end{array}$ \\
\hline 56.52 & 84.15 & 19 & 02 & 2014 & 0.05 & 0.56 & 28.13 & 30.00 \\
57.10 & 83.90 & 19 & 02 & 2014 & 0.05 & 0.56 & 28.13 & 30.00 \\
57.33 & 83.93 & 19 & 02 & 2014 & 0.05 & 0.56 & 28.13 & 30.00 \\
58.07 & 82.82 & 19 & 02 & 2014 & 0.05 & 0.56 & 28.13 & 30.00 \\
67.90 & 74.80 & 25 & 02 & 2014 & 0.05 & 0.56 & 28.13 & 30.00 \\
67.93 & 75.08 & 25 & 02 & 2014 & 0.05 & 0.56 & 28.13 & 30.00 \\
67.77 & 75.50 & 25 & 02 & 2014 & 0.05 & 0.56 & 28.13 & 30.00 \\
67.62 & 75.90 & 25 & 02 & 2014 & 0.05 & 0.56 & 28.13 & 30.00 \\
67.53 & 76.17 & 25 & 02 & 2014 & 0.05 & 0.56 & 28.13 & 30.00 \\
67.40 & 76.35 & 25 & 02 & 2014 & 0.05 & 0.56 & 28.13 & 30.00 \\
67.25 & 76.43 & 25 & 02 & 2014 & 0.05 & 0.56 & 28.13 & 30.00 \\
66.80 & 76.40 & 25 & 02 & 2014 & 0.05 & 0.56 & 28.13 & 30.00 \\
65.98 & 77.67 & 25 & 02 & 2014 & 0.05 & 0.56 & 28.13 & 30.00 \\
65.78 & 78.17 & 25 & 02 & 2014 & 0.05 & 0.56 & 28.13 & 30.00 \\
65.70 & 78.02 & 25 & 02 & 2014 & 0.05 & 0.56 & 28.13 & 30.00 \\
63.80 & 75.55 & 26 & 02 & 2014 & 0.05 & 0.56 & 28.13 & 30.00 \\
63.80 & 75.57 & 28 & 02 & 2014 & 0.05 & 0.56 & 28.13 & 30.00 \\
65.38 & 77.75 & 26 & 02 & 2014 & 0.05 & 0.56 & 28.13 & 30.00 \\
64.28 & 75.73 & 26 & 02 & 2014 & 0.05 & 0.56 & 28.13 & 30.00 \\
63.80 & 75.57 & 28 & 02 & 2014 & 0.05 & 0.56 & 28.13 & 30.00 \\
63.82 & 75.57 & 01 & 03 & 2014 & 0.05 & 0.56 & 28.13 & 30.00 \\
61.48 & 74.25 & 03 & 03 & 2014 & 0.05 & 0.56 & 28.13 & 30.00 \\
60.50 & 76.97 & 04 & 03 & 2014 & 0.05 & 0.56 & 28.13 & 30.00 \\
65.08 & 41.11 & 16 & 03 & 2015 & 0.03 & 0.09 & 12.60 & 3.00 \\
66.53 & 33.13 & 19 & 03 & 2015 & 0.14 & 0.09 & 50.40 & 3.10 \\
66.53 & 33.13 & 19 & 03 & 2015 & 0.56 & 0.09 & & 1.00 \\
\hline
\end{tabular}




\begin{tabular}{|c|c|c|c|c|c|c|c|c|}
\hline 66.55 & 33.03 & 20 & 03 & 2015 & 0.14 & 0.09 & 12.60 & 1.00 \\
\hline 66.53 & 33.03 & 21 & 03 & 2015 & 0.06 & 0.09 & 5.40 & 0.75 \\
\hline 66.53 & 33.12 & 22 & 03 & 2015 & 0.10 & 0.09 & 9.00 & 0.50 \\
\hline 66.53 & 33.08 & 23 & 03 & 2015 & 0.09 & 0.09 & 8.10 & 0.75 \\
\hline 66.53 & 33.13 & 23 & 03 & 2015 & 0.04 & 0.09 & 3.15 & 0.95 \\
\hline 66.52 & 33.08 & 24 & 03 & 2015 & 0.08 & 0.09 & 7.20 & 0.75 \\
\hline 66.53 & 33.05 & 24 & 03 & 2015 & 0.07 & 0.09 & 6.30 & 1.00 \\
\hline 66.55 & 33.08 & 25 & 03 & 2015 & 0.04 & 0.09 & 3.60 & 1.25 \\
\hline 64.53 & 38.85 & 29 & 02 & 2016 & 0.05 & 0.09 & 4.50 & 0.25 \\
\hline 65.08 & 41.11 & 29 & 02 & 2016 & 0.15 & 0.09 & 13.50 & 0.50 \\
\hline 66.55 & 33.14 & 01 & 03 & 2016 & 0.05 & 0.09 & 4.50 & 0.25 \\
\hline 66.54 & 33.05 & 02 & 03 & 2016 & 0.05 & 0.09 & 4.50 & 0.25 \\
\hline 64.32 & 40.79 & 03 & 03 & 2016 & 0.28 & 0.09 & 25.20 & 0.25 \\
\hline 64.32 & 40.78 & 03 & 03 & 2016 & 0.30 & 0.09 & 27.00 & 0.25 \\
\hline 64.32 & 40.79 & 03 & 03 & 2016 & 0.17 & 0.09 & 15.30 & 0.50 \\
\hline 64.32 & 40.79 & 03 & 03 & 2016 & 0.19 & 0.09 & 17.10 & 0.50 \\
\hline 64.32 & 40.79 & 03 & 03 & 2016 & 0.15 & 0.09 & 13.50 & 0.50 \\
\hline 66.55 & 33.10 & 04 & 03 & 2016 & 0.05 & 0.09 & 4.50 & 0.25 \\
\hline 66.54 & 33.15 & 06 & 03 & 2016 & 0.05 & 0.09 & 4.50 & 0.25 \\
\hline 72.26 & 68.82 & 09 & 04 & 2016 & 0.45 & 0.01 & 3.53 & 1.05 \\
\hline 72.94 & 65.36 & 22 & 04 & 2016 & 0.80 & 0.01 & 6.28 & 2.35 \\
\hline 78.45 & 70.89 & 23 & 04 & 2016 & 0.25 & 0.01 & 1.96 & 0.86 \\
\hline 78.70 & 67.47 & 23 & 04 & 2016 & 0.45 & 0.01 & 3.53 & 1.57 \\
\hline 69.93 & 71.78 & 28 & 04 & 2016 & 0.19 & 0.01 & 1.49 & 0.43 \\
\hline 71.76 & 71.51 & 29 & 04 & 2016 & 0.09 & 0.01 & 0.71 & 0.19 \\
\hline 71.44 & 71.09 & 29 & 04 & 2016 & 0.19 & 0.01 & 1.49 & 0.52 \\
\hline 71.28 & 69.75 & 30 & 04 & 2016 & 0.09 & 0.01 & 0.71 & 0.41 \\
\hline 69.81 & 69.84 & 01 & 05 & 2016 & 0.07 & 0.01 & 0.55 & 0.47 \\
\hline
\end{tabular}


Table S 2. $E C_{C_{3}^{2-}}^{c o r r}$ to $E C$ ratio (Mean $\pm \mathrm{SD}$; Min - Max), showing overestimation of $E C$ due to $E C_{\mathrm{CO}_{3}^{2-}}$ in the filtered snow samples.

\begin{tabular}{|c|c|c|c|}
\hline & 2014 & 2015 & 2016 \\
\hline$E C$ & $66 \pm 107$ & $87 \pm 36$ & $63 \pm 45$ \\
\hline$\left(\mu \mathrm{g} \mathrm{L}^{-1}\right)$ & $4-476$ & $49-153$ & $8-178$ \\
\hline$E C_{\mathrm{CO}_{3}^{2-}}^{\mathrm{Corr}}$ & $40 \pm 50$ & $83 \pm 37$ & $56 \pm 39$ \\
\hline$\left(\mu \mathrm{g} \mathrm{L}^{-1}\right)$ & $3-219$ & $46-152$ & $7-161$ \\
\hline$T C$ & $949 \pm 1258$ & $955 \pm 428$ & $1469 \pm 1278$ \\
\hline$\left(\mu \mathrm{g} \mathrm{L}^{-1}\right)$ & $50-5927$ & $550-1827$ & $226-4504$ \\
\hline $\mathrm{TC}_{\mathrm{CO}_{3}^{2-}}^{\mathrm{Corr}}$ & $865 \pm 1146$ & $949 \pm 428$ & $1449 \pm 1263$ \\
\hline$\left(\mu \mathrm{g} \mathrm{L}^{-1}\right)$ & $48-5580$ & $546-1864$ & $211-4493$ \\
\hline$E C_{\mathrm{CO}_{3}^{2-}}^{\mathrm{Corr}} / \mathrm{TC}_{\mathrm{CO}_{3}^{2-}}^{\mathrm{Corr}}$ & $5.3 \pm 2.4$ & $9.1 \pm 2.5$ & $5.7 \pm 3.8$ \\
\hline$(\%)$ & $2-12$ & $6-14$ & $1-15$ \\
\hline$E C_{C O_{3}^{2-}}^{\mathrm{corr}} / E C$ & $77 \pm 16$ & $94 \pm 6$ & $90 \pm 6$ \\
\hline$(\%)$ & $31-100$ & 77-99 & $76-100$ \\
\hline $\mathrm{CO}_{3}^{2-}$ & $84 \pm 191$ & $6 \pm 6$ & $20 \pm 32$ \\
\hline$\left(\mu \mathrm{g} \mathrm{L}^{-1}\right)$ & $0-753$ & $2-21$ & $1-119$ \\
\hline
\end{tabular}




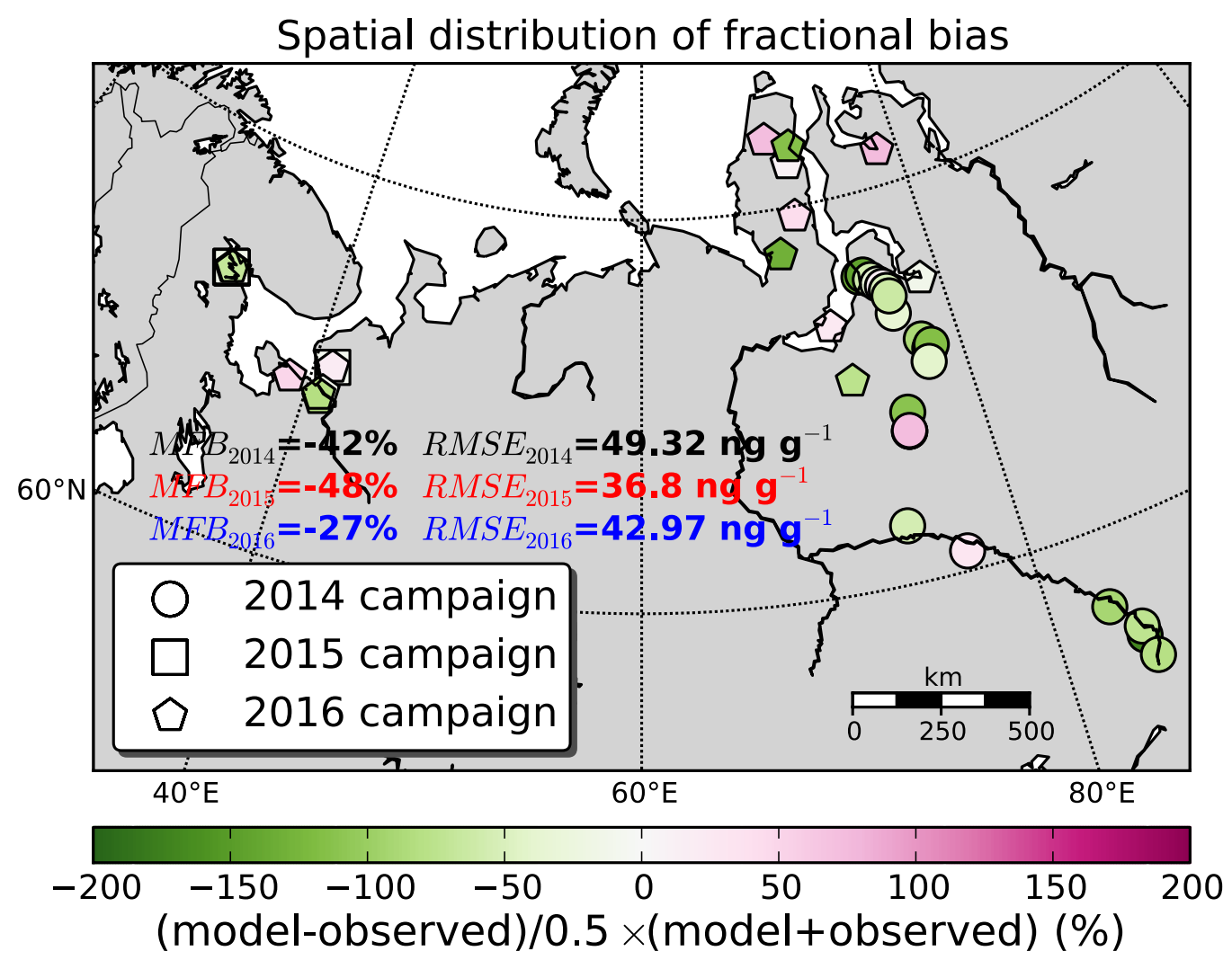

Figure S 1. Fractional bias $\left(F B=\left[\left(C_{m}-C_{o}\right) /\left(C_{m}+C_{o}\right) \times 0.5\right] \times 100 \%\right)$ for all samples collected from the three campaigns in Western Siberia and northwestern European Russia in 2014, 2015 and 2016. MFB (mean fractional bias) is the fractional bias averaged for all snow samples from 2014, 2015 and 2016, whereas RMSE is the root mean square error in $\mathrm{ng} \mathrm{g}^{-1}$ ). 


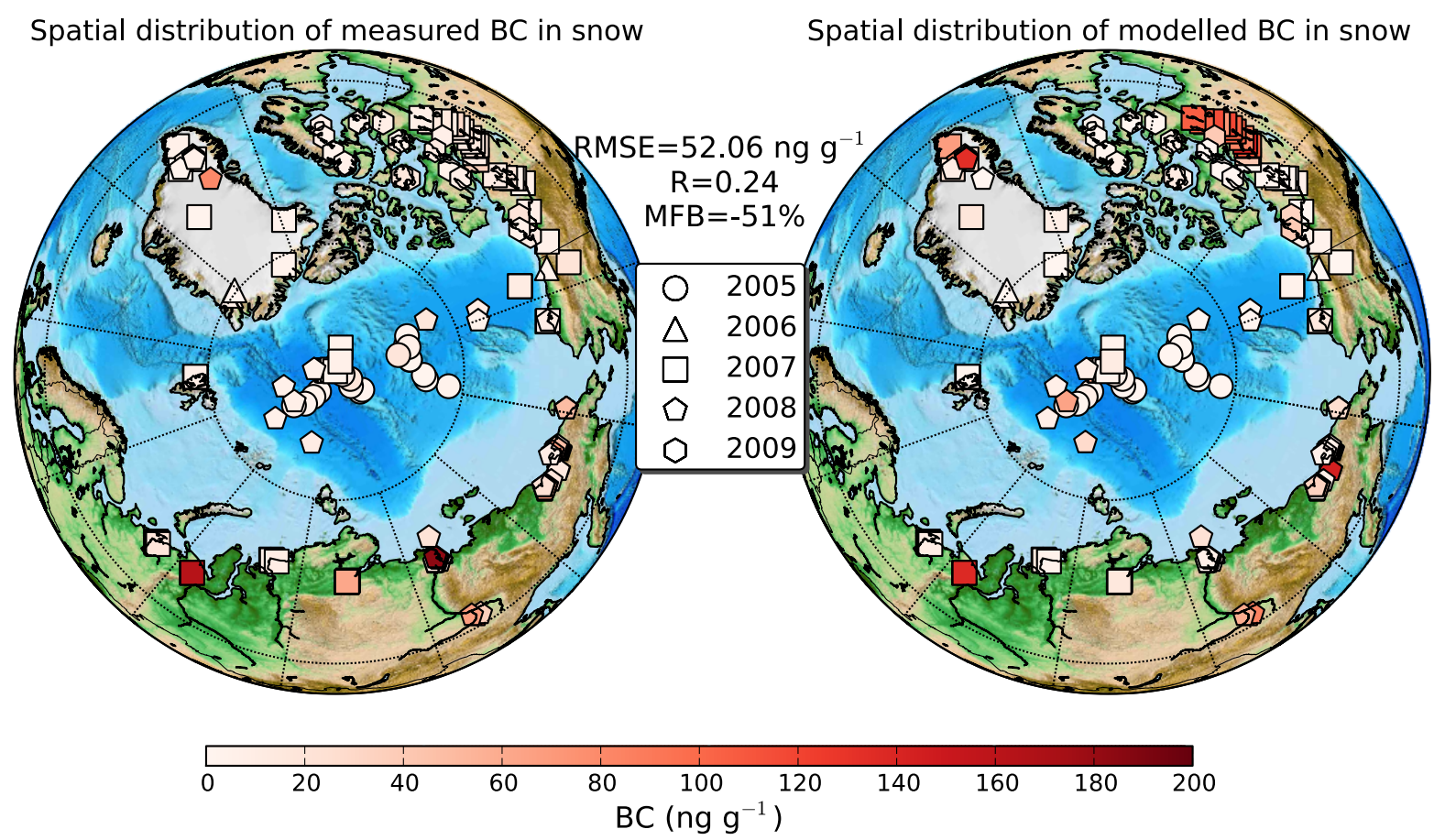

Figure S 2. Distribution of snow measurements of BC adopted from Doherty et al. (2010) in the Arctic from 2005 to 2009 (left). Simulated (FLEXPART) BC concentrations in snow for the same period (right). MFB, RMSE and correlation coefficient (R) values are further given. 
Comparison with MacDonald et al. (2016)

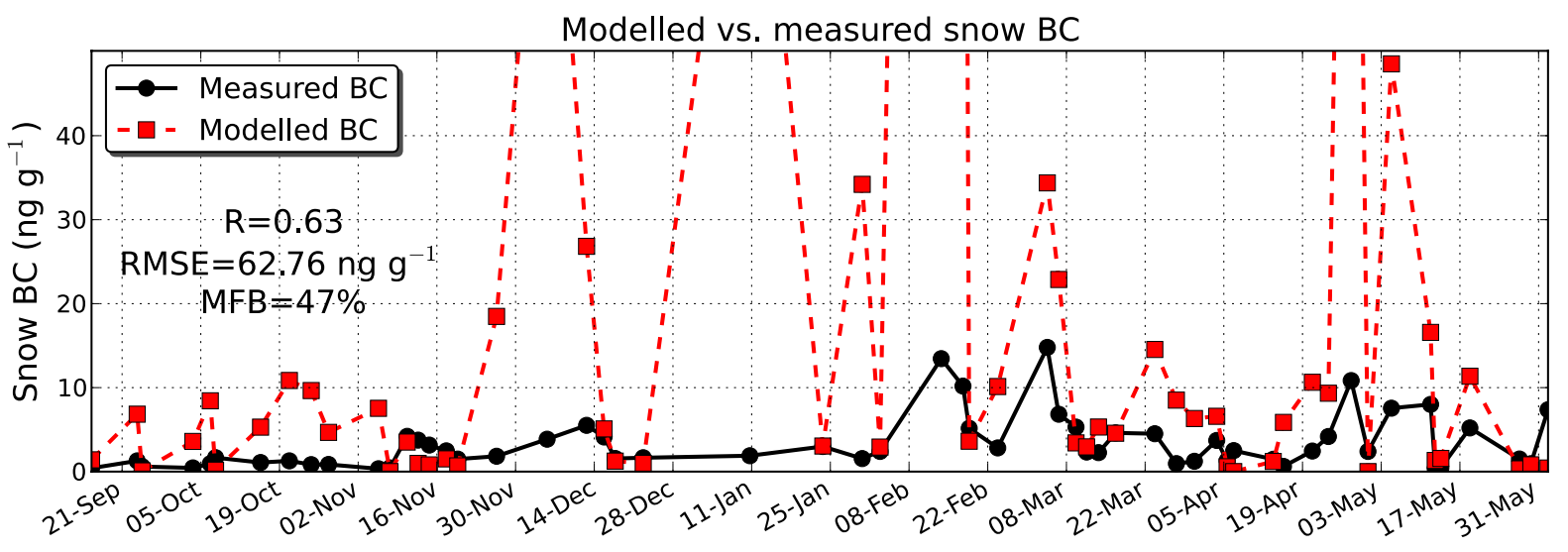

Figure S 3. Timeseries of simulated and measured BC concentrations in snow collected in Alert (Macdonald et al., 2016). Correlation coefficient (R) between modelled and measured $\mathrm{BC}, \mathrm{RMSE}$ and MFB values are also shown. 

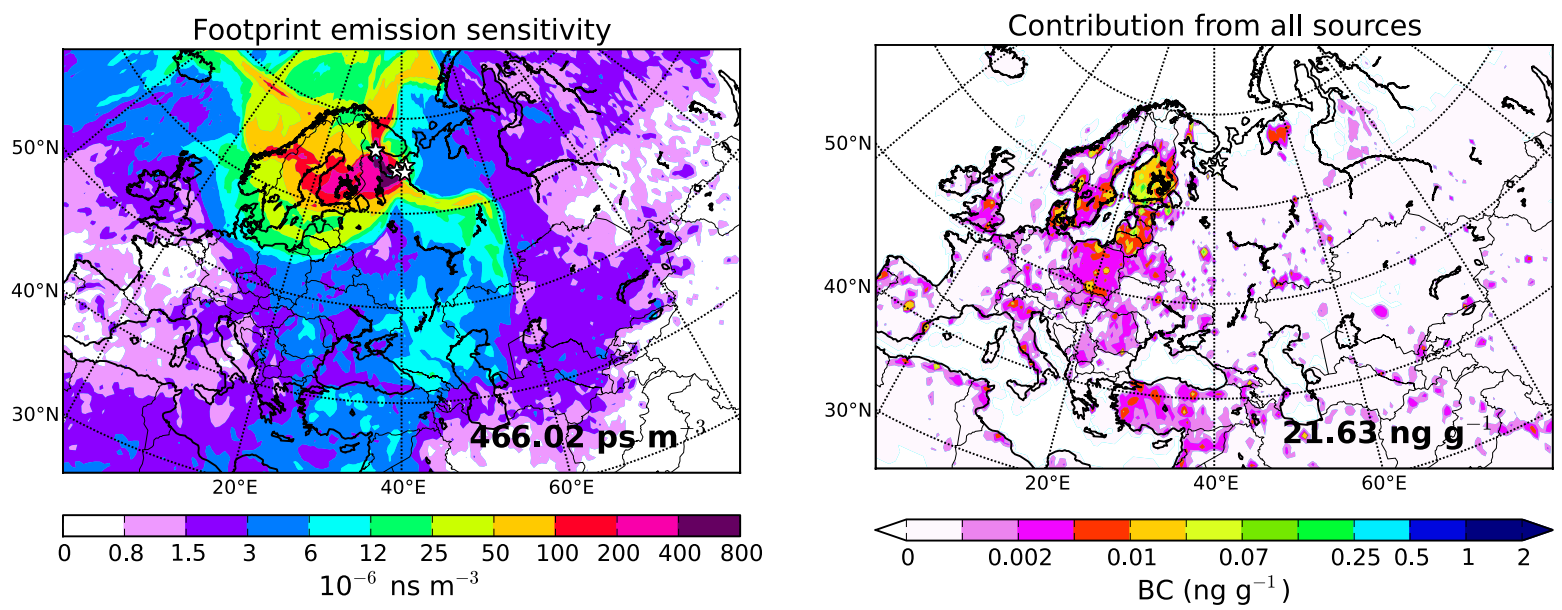

Contribution from transportation (TRA)
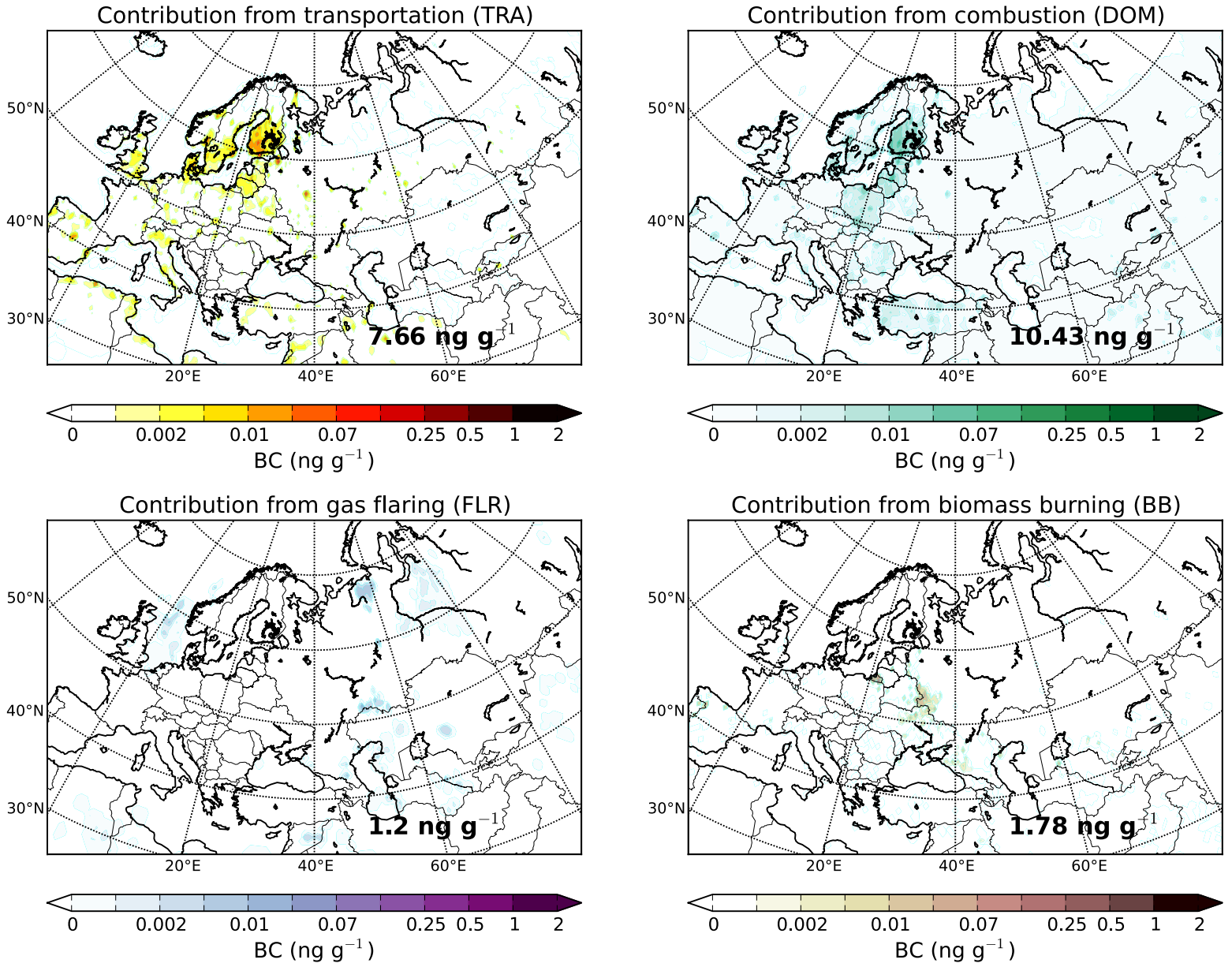

Figure S 4. Average footprint emission sensitivity and source contribution (from all sources,

TRA, DOM, FLR and BB) for all the samples located in northwestern European Russia. 

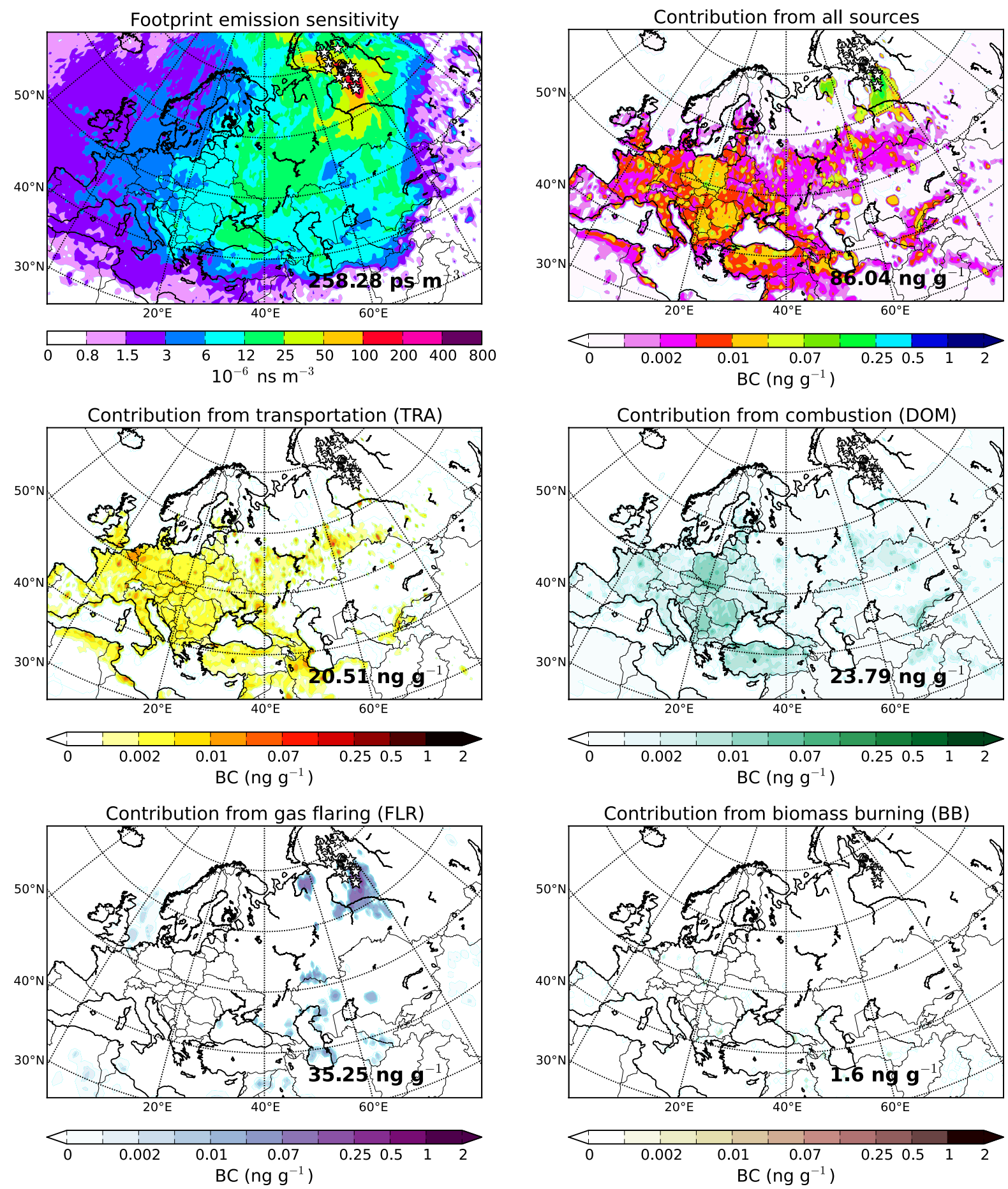

Figure S 5. Average footprint emission sensitivity and source contribution (from all sources, TRA, DOM, FLR and BB) for all the samples located in Western Siberia (north of $62^{\circ} \mathrm{N}$ ). 

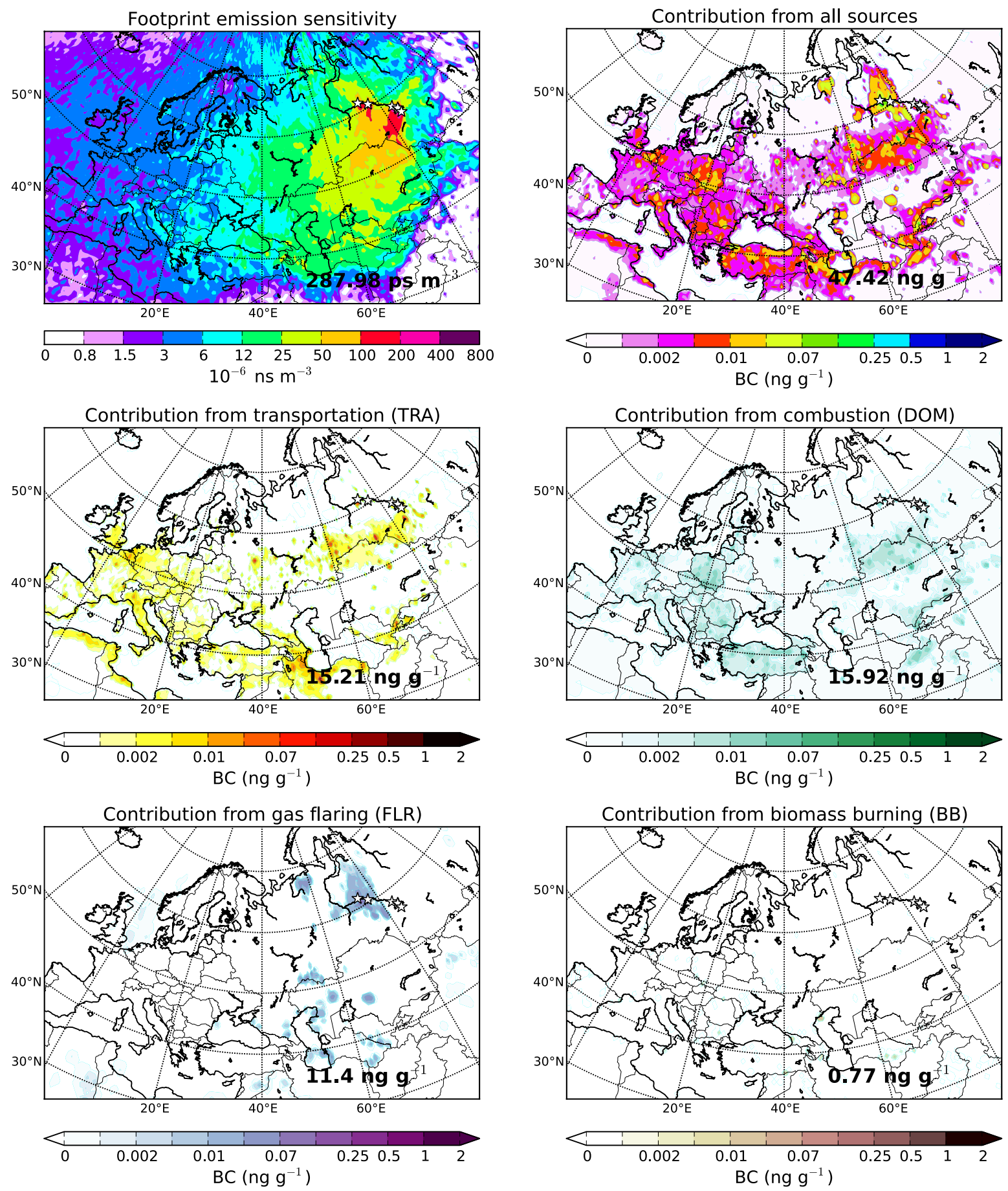

Figure S 6. Average footprint emission sensitivity and source contribution (from all sources, TRA, DOM, FLR and BB) for all the samples located in Western Siberia (south of $62^{\circ} \mathrm{N}$ ). 


\section{References}

Cavalli, F. et al., 2010. Toward a standardised thermal-optical protocol for measuring atmospheric organic and elemental carbon: the EUSAAR protocol. Atmospheric Measurement Techniques, 3(1), pp.79-89.

Chow, J. C., Watson, J. G., Pritchett, L. C., Pierson, W. R., Frazier, C. A., Purcell, R. G., 1993. The DRI Thermal/Optical Reflectance Carbon Analysis System: Description, Evaluation and Applications in U.S. Air Quality Studies. Atmospheric Environment, 27A(8), pp.1185-1201.

Doherty, S.J. et al., 2010. Light-absorbing impurities in Arctic snow. Atmospheric Chemistry and Physics, 10(23), pp.11647-11680.

Fagerli, H. et al., 2015. Transboundary particulate matter, photo-oxidants, acidifying and eutrophying components,

Gunasekaran, S. \& Anbalagan, G., 2007. Spectroscopic characterization of natural calcite minerals. Spectrochimica Acta - Part A: Molecular and Biomolecular Spectroscopy, 68(3), pp.656-664.

Jankowski, N. et al., 2008. Comparison of methods for the quantification of carbonate carbon in atmospheric PM10 aerosol samples. Atmospheric Environment, 42(34), pp.80558064. Available at: http://dx.doi.org/10.1016/j.atmosenv.2008.06.012.

Macdonald, K.M. et al., 2016. Observations of Atmospheric Chemical Deposition to High Arctic Snow. Atmospheric Chemistry and Physics Discussions, (November), pp.1-19. Available at: http://www.atmos-chem-phys-discuss.net/acp-2016-944/.

Subramanian $\uparrow$, R., Khlystov, A.Y. \& Robinson, A.L., 2006. Effect of Peak Inert-Mode Temperature on Elemental Carbon Measured Using Thermal-Optical Analysis. Aerosol Science and Technology, 40(10), pp.763-780.

Wallén, A., Lidén, G. \& Hansson, H.-C., 2010. Measured elemental carbon by thermo-optical transmittance analysis in water-soluble extracts from diesel exhaust, woodsmoke, and ambient particulate samples. Journal of occupational and environmental hygiene, 7(1), pp.35-45.

Yttri, K.E. et al., 2007. Elemental and organic carbon in $\mathrm{PM}_{10}$ : a one year measurement campaign within the European Monitoring and Evaluation Programme EMEP. Atmospheric Chemistry and Physics, 7(22), pp.5711-5725. Available at: http://www.atmos-chem-phys.net/7/5711/2007/acp-7-57112007.html\%5Cnhttp://www.atmos-chem-phys.net/7/5711/2007/. 